\title{
Innovative functional products development as an element for the Russian Federation population health preservation
}

\author{
Natalia Zavorokhina*, Ekaterina Minnihanova, Olga Feofilaktova, and Olga Chugunova \\ Ural State University of Economics, 8 March St., 62, 620144 Yekaterinburg, Russian Federation
}

\begin{abstract}
The agricultural development priorities and the agro-industrial complex for the Russian Federation Food Security Doctrine implementation are considered. It was determined that, among other things, the development of new specialized and functional food products was included in the list of priority areas of fundamental scientific research for 2021-2030. It has been shown that the population of the Russian Federation with low-calorie food products, which are based on domestic agricultural raw materials, meets the interests of the state as a whole: about $12 \%$ of the total health care budget in the world is spent on solving the problem of diabetes mellitus, in the Russian Federation - 569 billion rubles a year. The main trends in the development of innovative functional products presented by various researchers are systematized. The authors consider innovative functional products on the example of low-calorie meals from the public health viewpoint. The prospects for the use of sweeteners for the development of low-calorie sweet dishes for the diabetes prevention are assessed. Experimental data on the selection of the optimal combination of sweeteners are presented. Optimal characteristics are possessed by a mixture of aspartame-sodium saccharinate-sucralose in a ratio of 3: 2: 1, which has a synergistic effect of $12 \%$. A complex supplement is proposed, including a mixture of Dolceta sweeteners and apple pectin. Low-calorie mass-market meals have been developed.
\end{abstract}

\section{Introduction}

One of the priorities for the development of the agro-industrial complex of the Russian Federation is the action plan for the implementation of the provisions of the Russian Federation Food Security Doctrine, approved by the order of the Government of the Russian Federation dated June 9, 2020 No. 1516-r. According to the roadmap, an action plan was approved that covers the mechanisms of sufficient production of food products, including their economic accessibility, provision of the general population with healthy food products necessary for an active and healthy lifestyle. At the same time, according to the Order of the Government of the Russian Federation of December 31, 2020 No. 3684-r "On the approval of the Program of fundamental scientific research in the Russian Federation for a long-term period", the "development of innovative technologies new

\footnotetext{
${ }^{*}$ Corresponding author: ip@usue.ru
} 
specialized and functional food products, food ingredients". Thus, the state mechanisms of economic development of the agro-industrial complex, the consumer market of wholesome nutrition, where the outline is the development of new technologies, technical solutions, aimed primarily at human health, are identified.

The most important task of agriculture and the agro-industrial complex as a whole is not only to ensure the food security of the country or the targeted population of the Russian Federation with a sufficient amount of environmentally friendly, high-quality agricultural products, but also to analyze the impact of produced agricultural raw materials on the health of the population.

If we look at the social problem of health preservation of the population from the point of view of the economy, then a statistical analysis shows that deaths and illnesses of the able-bodied, economically active population of the Russian Federation lead to losses of about 1.5 trillion rubles a year. First of all, these losses are associated with lost products, sick leaves. The correlation between public health, labor productivity and the economic component is obvious [1]. With a multitude of socially significant diseases of the population of the Russian Federation, the greatest concern is the spread of diabetes mellitus.

Statistical data analysis shows that about $12 \%$ of the total health care budget is spent on solving the problem of diabetes mellitus around the world, while in the Russian Federation these costs amount to about 569 billion rubles per year, which corresponds to $1 \%$ of the total Russian Federation gross domestic product [2].

The WHO report for 2019 indicates that the main causes of diseases in the population of developed European countries are alcohol consumption - $8.4 \%$, overweight $-4.8 \%$, low consumption of vegetables and fruits $-3.5 \%$. Among them, obesity and diabetes mellitus are the most common diseases of the human endocrine system.

To assess the state of food security, a system of indicators is used, which includes, among other things, the daily caloric intake of a person: the amount of proteins, fats, carbohydrates, vitamins, micronutrients consumed by a person per day. The production and attraction of additional agricultural raw materials with high nutritional and biological value to the country's food turnover is an urgent scientific direction. Scientific interest is also associated with the production of raw materials and complex food systems for the prevention of such socially significant diseases as obesity and type 2 diabetes [3].

Providing the population of the Russian Federation with low-calorie food products, which are based on domestic agricultural raw materials, meets the interests of the state as a whole.

Low-calorie functional foods are foods in which the calorie content is reduced by more than $30 \%$, while the nutritional value is preserved. Traditionally, in dietary nutrition, they reduce the calorie content of foods by lowering the content of high-calorie fats and easily digestible simple sugars. Speaking about the raw material market for low-calorie functional foods, it should be remembered that the main low-calorie foods are primarily agricultural crops, vegetables, fruits, which contain a significant amount of useful fiber, pectins, vitamins and micronutrients.

There is growing concern that the consumption of free sugars increases the total caloric intake of the diet, its glycemic index and reduces the consumption of foods with high nutritional value, which inevitably leads to such undesirable health consequences as obesity, diabetes mellitus, atherosclerosis, and allergic reactions [4].

The popularity of such food product categories as flour culinary and confectionery products, sweet dishes remains quite high all over the world. It should be noted that the inclusion of low-calorie components in the recipes for sweet dishes often leads to a deterioration in organoleptic characteristics in comparison with high-calorie sweet dishes. When replacing in the composition of recipes with low-calorie components, taste characteristics decrease, unpleasant extraneous aftertastes may appear, and the degree of 
pleasure that a consumer receives from the consumption of sweet dishes decreases. The problems of reducing the calorie content of food rations, the creation of innovative technologies for the production of specialized and functional foods are discussed annually at the All-Russian Congress of Nutritionists and Nutritionists. The main trends in the development of innovative functional products presented by various researchers are presented below.

The authors of Gendelek G.F., Gendelek A.M. investigated the use of sugar substitutes and high-intensity sweeteners in diet therapy of diabetes mellitus and obesity.

Authors Nepovinnykh N.V., Ptichkina N.M. developed a recipe for low-calorie jelly based on curd whey and fruit juices. The authors replaced starch with polysaccharide thickeners, sugar-non-carbohydrate sweeteners, gave a comparative assessment of the qualitative characteristics of the developed jelly, analyzing the change in organoleptic and physicochemical parameters depending on the amount of low-carbohydrate components introduced.

Abramova Yu.P., Dolgorukova M.V. studied the possibility of using sugar substitutes, in particular, sorbitol, in the production of creamy desserts such as "Panacotta" in order to reduce calorie content is considered.

Krylova E.N., Mavrina E.N., Savenkova T.V. analyzed the use of sweeteners in the production technology of jelly marmalade based on gelatin. Isomalt polyol was used as a sweetener, as it is the most technologically advanced, heat-stable, low-calorie polyol. The authors chose gelatin as a gelling agent, since it is used for the production of confectionery products of a special group - with chewing properties that have an elastic-plastic consistency.

Morozova S.S., Bakumenko O.E., Tarasova V.V. developed formulations of food jelly concentrates using sweeteners and intense sweeteners such as xylitol, fructose, stevioside, maltitol.

Popov V.S., Kalimova Yu.M. worked out recipes for products made from low-calorie biscuit dough by including oat flour and sweeteners - stevia and erythritol - which can be used as a base for some desserts.

G.V. Ivanova studied new types of dietary supplements in the production of sweet dishes for dietary and therapeutic-prophylactic nutrition. The possibility of using decoctions of Icelandic cetraria (CI) as a biologically active additive (BAA) in the preparation of sweet whipped products, mousses, creams, as well as jelly, jelly, was investigated, which made it possible to increase the nutritional value of sweet dishes, but slightly reduce their calorie content.

E.Yu. Wolf studied the effect of low-carbohydrate thickening polysaccharides and fructose on the texture and flavor of cranberry mousse. The author assessed the influence of these components on foam stability and churning ability, presented data on nutritional value, and developed a technology for the production of this mousse at the enterprise.

I.V. Maceichik, A.N. Kudryashova developed formulations and technology for the production of frozen whipped desserts for functional purposes using the stevioside sweetener.

Amyaga B.V., Barsukova N.V. created a recipe for the low-calorie cheese-curd dessert "Tiramisu" for specialized nutrition with the use of stevioside.

V. A. Gerasimova, E. S. B

\section{Materials and methods}

For the development of low-calorie sweet dishes, the following types of raw materials were used: sweeteners that meet the requirements of TR CU 029/2012 (aspartame, sodium cyclamate, sodium saccharinate, sucralose, stevioside and their solutions corresponding to a 
5\% sucrose solution according to the calculated sweetness coefficient of sweeteners); polysaccharides (agar-agar according to GOST 16280, sodium alginate according to TU 9284-030-00462769-06, gelatin according to GOST 11293-89; pectin according to GOST 29186-91, lecithin according to GOST 32052-2013; magnesium alginate and sodium alginate according to GOST 33310 -2015; food acids (citric monohydrate according to GOST 908-2004; lactic according to GOST 490-2006, sucrose according to GOST 634175 ); sucrose solutions: $2 \%, 5 \%, 7 \%$. The control sample was a $7.5 \%$ sucrose solution; triple mixtures of sweeteners (triads) with their equivalent content, which correspond to $5 \%$ sucrose concentration. When developing the basic recipe for low-calorie meals, sodium citrate was used according to GOST standard 31227-2004, calcium citrate according to GOST R 54538-2011

To calculate the nutritional and energy value, we used the automated "Computer program for modeling the recipes of low-calorie food products (NK-2020")", developed by the authors.

For the experiment, the following research methods were used: the organization of the tasting analysis corresponded to GOST ISO 6658-2016 "Organoleptic analysis. Methodology. General guidance ", GOST ISO 10399-2015 Organoleptic analysis. Methodology. Test "duo-trio"; GOST 31986-2012 Catering services. The method of organoleptic assessment of the quality of public catering products; GOST ISO 11036-2017 Organoleptic analysis. Methodology. Structure characteristics; GOST ISO 8588-2011 Organoleptic analysis. Methodology. Tests "A" - "Not A"

\section{Results and discussion}

The main task of developers of functional products is a specified properties set formation on the one hand, the product should be massive, meet the theory of adequate nutrition, have a high nutritional value, on the other hand, it should be comparable in taste to a traditional high-calorie product and at the same time have a low cost price. Therefore, it is extremely important to select sugary low-calorie raw materials that correspond to the sugar profile [5].

To develop formulations of innovative functional products for persons with diabetes mellitus, raw materials with a low glycemic index were used, including sweeteners. In the European Union (EU), the use of sweeteners is regulated by the European Directive 94/35 / EC. According to it, eight intense sweeteners are allowed for use: acesulfame K, aspartame, aspartame, acesulfame salt, cyclamate, neohesperidin, saccharin, sucralose and thaumatin [6-9]. In the Russian Federation, the use of sweeteners is currently standardized by TR CU $029 / 2012$.

At the first stage, in order to form the optimal flavor of sweet dishes, the taste and aftertaste of sweeteners and their combinations were analyzed. The choice of sweeteners is due to their solubility, manufacturability, availability and wide representation on the Russian market. To determine the optimal flavor and solubility, a comparative tasting of sweetener solutions was carried out, the calculated sweetness of which corresponded to a $7.5 \%$ sucrose solution in comparison with the standard (7.5\% sucrose solution). It was determined that the most soluble is sodium saccharinate $(660 \mathrm{~g} / 1)$, the least soluble is aspartame (100 g / 1); acesulfame potassium, cyclamate and sucralose have an average solubility of 270, 200, $282 \mathrm{~g} / 1$, respectively.

At the second stage, the synergism of sweeteners in ternary mixtures was investigated, from which solutions with an estimated sweetness of $7.5 \%$ by glucose were prepared. The choice of the ingredients of the ternary mixtures was due to the known literature data on the synergistic effect of these sweeteners in binary combinations. Using the duo-trio similarity test, namely the constant reference technique applied when one product is known to the prover, the degree of difference between the sweet taste of sucrose and the selected triads of 
sweeteners was investigated. To achieve a high statistical likelihood of similarity / difference, an $\alpha$-risk of 0.01 was chosen. In the course of the tastings, where the tasters were testers with proven sensory sensitivity, the optimal triad (a mix of 3 sweeteners) was determined, which has the optimal taste and aftertaste and the highest synergism aspartame-saccharinate-sucralose. The lowest tasting points were scored by a mixture of sweeteners aspartame-acesulfame-saccharinate, which has a long, bitter-metallic aftertaste. Statistical processing of the results showed that with a $99 \%$ probability, not less than $11.1 \%$, a person will distinguish a sample of aspartame-saccharinate-sucralose in comparison with a control sucrose solution, which indicates a high similarity of this sample with the standard sucrose solution.

The calculated coefficient of sweetness of the triad of aspartame-saccharinate-sucralose hereinafter referred to as the "Dolcetta" mixture as 3: 2: 1 is 360 units. The resulting mixtures of sweeteners have a synergistic effect, that is, there is a mutual enhancement of sweetness by $12 \%$, which makes it possible to reduce the cost of the developed sweet dish [10]. The prime cost of the mixture is 2240 rubles $/ \mathrm{kg}$, which is 8 times cheaper than using an equivalent amount of sugar. For the developed mixture of sweeteners, TU 10.89.19.150016-02069214-2019 "Dolchett's sweetener mixture" has been developed. In terms of microbiological and safety indicators, the Dolcetta sweetener mixtures comply with TR CU 029/2012.

When selecting thickeners and gelling agents, the aftertaste longitude and kinematic viscosity of the polysaccharide solution were assessed: agar-agar, pectin, gums and magnesium and sodium alginate. It has been determined that agar-agar, despite good gelling, in combination with a mixture of sweeteners gives a short aftertaste; citrus highly esterified pectin, despite a long aftertaste (60 caudal), has a tart citrus note, which limits its use in combination with berry or milky flavors; guar and xanthan gums give a soapy flavor, transforming the apple flavor; sodium alginate, having a high kinematic viscosity and a long-term indifferent aftertaste, is still inferior to apple pectin in terms of a set of indicators [11]. Apple pectin contains a small amount of calories, is high in dietary fiber, lengthens the aftertaste, is dissolved without forming lumps, which is an important technological factor in the preparation of sweet dishes. The use of pectin makes it possible to add dietary fiber to the recipes of this category of dishes, which reduces the risks of developing obesity, type 2 diabetes, and some oncological diseases. As a result of the research, a dry mix recipe was developed (Table 1), which can be the basis for the development of low-calorie sweet dishes and has variability in use depending on the taste and aroma characteristics of the raw materials used and its corrective ability.

Table 1. Low Calorie Sweet Base Mix Recipe

\begin{tabular}{|c|c|}
\hline Component of the mixture & \% wt. \\
\hline Low esterified apple pectin & 85,863 \\
\hline $\begin{array}{c}\text { Complex additive of } \\
\text { sweeteners "Dolcetta" }\end{array}$ & 1,639 \\
\hline Calcium citrate & 7,636 \\
\hline Sodium citrate & 4,863 \\
\hline Total & 100,00 \\
\hline
\end{tabular}

This additive is introduced in the amount of 2-3 $\mathrm{g}$ per $100 \mathrm{~g}$ of ready-made sweet dish, and contains only 4.1 to $5.7 \mathrm{kcal}$, which can significantly reduce the energy value of the developed recipes.

Further, in accordance with the objectives of the study, we have developed formulations of low-calorie sweet dishes with a developed complex supplement. The assortment of sweet foods for those with diabetes included creams, jellies and mousses. Cream recipes with 
sugar and gelatin were selected as control formulations for comparing nutritional and biological values.

It should be noted that the minimum thermal effect on the product during the preparation of non-calorie sweet dishes contributes to the preservation of mass, food nutrients, and also allows you to obtain a product with high organoleptic characteristics. The complex additive was prepared as follows: the required amount was poured with water at room temperature and left to swell for 25-30 minutes. Vegetable raw materials were processed in accordance with the technological recommendations for processing raw materials, blanched in a combi-steamer in the "Steam" mode, cooled to room temperature, then ground to a puree state in a blender at $30,000 \mathrm{rpm}$, wiped in a pulper, cooled to a temperature of 20-250C. Vanillin was dissolved in warm water until completely dissolved, filtered. For each name of the cream, 3 variants were developed with varying the amount of flavoring components (a total of 12 model samples). The tasting results showed that the developed samples have a high score for all indicators. Apple pectin in the developed recipes of low-calorie dishes is a prebiotic, plays the role of a gelling agent, as well as prolongs the aftertaste and enhances the aroma of the developed creams.

The authors developed a technology for the preparation of these low-calorie sweet dishes based on fruit, vegetable and dairy raw materials, studied the structural and mechanical properties in comparison with recipes prepared according to the traditional recipe and technology based on gelatin and sugar (control). The research results showed that in the developed low-calorie sweet dishes there is no syneresis, they have a high thixotropy. The developed low-calorie dishes can be produced both at food industry enterprises and at public catering enterprises, which determines their massive consumption. Local zoned plant raw materials, cake and berry pomace, rich in fiber and P-active polyphenols, can be used as a fruit and vegetable component. The use of the developed sweet dishes will increase the functional reserves of the body and help maintain health and prevent diseases in healthy and conditionally healthy people, increase the nutritional and therapeutic value of diets in patients with non-infectious alimentary-dependent diseases, such as atherosclerosis, type 2 diabetes mellitus, obesity, diseases of the digestive system, as well as prevent oxidative stress.

\section{Conclusions}

The health of the nation is an important economic resource of society and the main condition for the reproduction of labor potential, it is an indicator of the people life quality. The United Nations has identified 17 Sustainable Development Goals that UN member states have agreed to achieve by 2030. Almost all goals (16 out of 17) are related to the health of the world's population while respecting the environment and combating climate change. The economic stability of a country depends on many factors, the most important of which is the positive dynamics of the development of agriculture and the agro-industrial sector, which depend, first of all, on the human factor - its qualifications, understanding of goals and, of course, health. State policy in the Russian Federation is consistent with the global goals of developed countries. However, the work of ensuring the health of the nation also faces dramatic changes, systemic problems and the challenge of social norms and influences [12,13]. Since agricultural producers provide the population with food, raw materials and materials for various spheres of human life, it can be confidently asserted that the most important sector of the economy, agriculture, is responsible for the health and life expectancy of the country's population. Miscalculations in agriculture, misuse of hazardous agricultural materials, contaminated food can lead to food shortages [14]. That is why an integrated approach, including the interconnection of agricultural enterprises, agricultural 
enterprises, developers of functional food products, including low-calorie ones, is an important task at this stage of development of the Russian Federation.

\section{References}

1. K.A. Samutin, Public health as an integral element of the state economic policy, 11 (109), 131 (2012)

2. A.E. Yuzhakova, A.A. Nelaeva, Yu.V. Khasanova, I.V. Medvedev, Nutrition issues, 89(6), 23 (2020)

3. P. Lou, P. Chen, L. Zhang., P. Zhang , J / Yu, N. Zhang, BMJ, 2, 956 (2012)

4. V.A. Kobrinskii, A.S. Kadykov, M.G. Poltavskaya, N.A. Blagosklonov, M.N. Kovelkova, Preventive Medicine, 22(5), 78 (2019)

5. O.V. Chugunova, N.V. Zavorokhina, The use of methods of tasting analysis in the modeling of food recipes with given consumer properties, 204 (2010)

6. M Bridel, Bull Soc Chem Biol., 13(7), 409 (2007)

7. A Gasmalla, Food and rev., 4(2), 2 (2014)

8. H Kohda, R. Kasai, K. Yamasaki, K. Murakami, O. Tanaka, Phytochem, 9(15), 981 (2001)

9. To Eckel-Mahan, P. Sassone-Corsi, Physiol. Rev, 93, 107 (2013)

10. G. R. Sridhar, N. S. Sanjana, World J. Diabetes, 7, 515 (2016)

11. N.V. Zavorokhina, O.V. Chugunova, E.Yu. Minnikhanov, Food industry, 9, 66 (2019)

12. E.Yu. Minnikhanova, N.V. Zavorokhina, A.A. Gilina, Food Industry, 5(2), 71 (2020)

13. P.P. Vasiliev, SKAGS, 326 (2015)

14. M Arbenz, D. Gould, C. Stopes., Organic Agriculture, 7(3), 199 (2017) 